ORIGINAL ARTICLE

\title{
The Role of Natural Medicines on Wound Healing: A Biomechanical, Histological, Biochemical and Molecular Study
}

\author{
Kotian Sushma ${ }^{1}$, Bhat Kumar ${ }^{2 *}$, Pai Sreedhara ${ }^{3}$, Nayak Jayakrishna ${ }^{4}$, Souza \\ Anne $^{1}$, Gourisheti Karthik ${ }^{2}$, Padma Divya ${ }^{1}$
}

\section{OPEN ACCESS}

Citation: Kotian Sushma, Pai Sreedhara, Nayak Jayakrishana, Souza Anne, Gourisheti Karthik, Padma Divya, Bhat Kumar. The Role of Natural Medicines on Wound Healing: A Biomechanical, Histological, Biochemical and Molecular Study. Ethiop J Sci.2018;28(6):759.

doi:http://dx.doi.org/10.4314/ejhs.v28i6.11 Received: June 02, 2018

Accepted: June 28, 2018

Published: November 1, 2018

Copyright: (C) 2018 Kotian S, et al. This is an open access article distributed under the terms of the Creative Commons Attribution License, which permits unrestricted use, distribution, and reproduction in any medium, provided the original author and source are credited.

Funding:

Competing Interests: The authors declare that this manuscript was approved by all authors in its form and that no competing interest exists. Affiliation and Correspondence:

${ }^{1}$ Department of Anatomy, Center for Basic Sciences, Kasturba Medical College Manipal, Manipal Academy of Higher Education, Manipal, Karnataka, India, ${ }^{2}$ Dept. of Anatomy, RAK College of Medical Sciences, RAK Medical \& Health Sciences University, Ras Al Khaimah, UAE ${ }^{3}$ Dept. of Pharmacology, Manipal College of Pharmaceutical Sciences, Manipal, Manipal Academy of Higher Education, Manipal, Karnataka, India, ${ }^{4}$ Sri Dharmasthala Manjunatheshwara Ayurvedic College, Udyavara, Udupi, India

*Email: kumarbhat@rakmhsu.ac.ae

\section{ABSTRACT}

BACKGROUND: Traditional medicinal systems like Ayurveda and Indian folk medicine have used Honey, Ghee, Glycyrrhiza glabra, and Nerium indicum effectively for treating wounds. The known result of these medications is faster healing. However, the mechanism of actions at the tissue level, the biochemical and molecular mechanisms of healing is not well explored and documented. This present study was therefore designed to study the efficacy of these traditional medicines singly and in combinations on excision wounds in Wistar rats.

METHODS: At two different intervals (i.e., day 8 and day 16), biomechanical, histological and immunohistochemical (IHC) parameters were assessed at the wound site. IHC focused on the inflammatory rate by evaluating the level of cytokine, IL $1 \beta$ and the tissue remodeling by studying the activity of myofibroblasts.

RESULTS: Rapid epithelization, better remodeling, favorable inflammatory changes and an adequate myofibroblast activity at the wound site was observed in all the treated groups compared to control.

CONCLUSION: This study is therefore useful in exploring the mechanism of action of these traditional medicines and providing valuable scientific evidence.

KEYWORDS: Wound Healing, Honey, Ghee, Glycyrrhiza glabra, Nerium indicum

\section{INTRODUCTION}

Every living tissue responds to injury. Tissue disruption, operative or traumatic, instigates a set of priorities in every organism. They include termination of bleeding, avoiding infection and refurbishment of the integrity and function of the tissue. This process of tissue repair is called wound healing. Wound healing has three major stages: an inflammatory phase wherein cytokines, growth factors and other inflammatory mediators are produced (1). The next phase of healing is the proliferation phase wherein cells like fibroblasts and endothelial cells are recruited. The last phase includes the production and reorganization of extracellular matrix leading to tissue repair and regeneration (1). 
Wounds have affected humans since pre-historic times, and the treatment of healing wounds is an art which is as old as humanity (2). Research on drugs helpful in wound healing is a rapidly growing area in biomedical sciences. The advancement in this field has enabled the production of various molecules related to the process of wound repair. However, the current trends in the wound management target only to avoid inflammation and growth of microbes at the site of the injury (2).

Regardless of finding new methods to enable the process of wound healing, wound care has found solace in the roots of medicine and is embracing some of the traditional therapies used ages ago. Ayurveda and folk medicinal systems encompass many such entities that traditionally accelerate healing. Healers yearn for traditional medicines for their high acceptability rate and real toleration (3).

Honey, Ghee and medicinal plants like Glycyrrhiza glabra (GG) and Nerium indicum (NI) are few of the chosen traditional medicines known for their wound healing benefits in Ayurveda and folk medicine. The efficacy of healing observed in the wounds treated with these traditional medicines shows promising results although the scientific evidence available in their defense are few. Several studies have demonstrated the role of individual application of honey (4) or ghee (5) in wound management.

GG is a plant of medicinal value. Being an herbaceous perennial legume, GG commonly inhabits Southern Europe and certain parts of Asia, such as India. It is an oldest and extensively used herb from the primeval times in Ayurveda. It is used as a medicine as well as a flavouring agent that masks the disagreeable taste of other medicinal preparations. They had also been used by the Egyptian, Chinese, Greek, Indian and Roman civilizations in various medicinal preparations. The wound healing, antiinflammatory, anti-ulcer and skin regeneration activity of GG is also documented by research (6). $\mathrm{NI}$ is another plant used in folk medicine to treat wounds. It is a native of the Mediterranean region, often cultivated as an ornamental shrub in gardens worldwide. NI is considered as one among the most poisonous garden plants that are toxic in all its parts. Although toxic, the plant is known for its benefits in folk medicine. NI has many medicinal properties. It serves as an astringent, anthelmintic, aphrodisiac, acrid, stomachic, febrifuge, diuretic, emetic, expectorant, cardiotonic, anti-cancerous drug, etc. It is used in the treatment of asthma, renal and vesicle calculi, chronic stomach and skin related problems, snake bites, joint pains, leprosy, cancer, ulcers, etc. (7).

Our previously published report on wound healing in incision wounds using these traditional medicines singly and in combinations provided valuable evidence on the microenvironmental changes in the wound site, thus proving the efficacy of these medications (8). However, the effect of these traditional medicines on the rate of wound closure and epithelialization in excision wounds is rarely discussed. Further, confirmatory quantitative findings on the inflammatory responses of the injury and the role of myofibroblasts in maintaining the skin homeostasis in the wounds treated with these traditional medicines are seldom available. Additionally, the impact of these drugs on the expression of the cytokines is also not experimented.

This study was therefore designed to scientifically evaluate the cellular mechanisms of wound healing by these medicinal preparations, i.e., honey, ghee and two medicinal plantsGlycyrrhiza glabra (GG) and Nerium indicum (NI). It was done singly and in combination in the excision wound model to provide quantitative evidences for the same. This study may also provide an understanding of the actual healing process, the mechanisms of which otherwise remains elusive.

\section{MATERIALS AND METHODS}

Test material-procurement and preparation: Unprocessed honey in its raw form, Cow's ghee, and roots of $G G$ were procured from Sri Dharmasthala Manjunatheshwara (SDM) Ayurvedic pharmacy, Udyavara, Udupi. Leaves of NI were collected in the month of December from its natural habitat in Udupi and was identified and authenticated by Dr. K. Gopal Krishna Bhat,

DOI: http://dx.doi.org/10.4314/ejhs.v28i6.2 
Professor of Botany (Rtd), Poornaprajna College, Udupi, Karnataka, India. A voucher specimen has been preserved in the Department of Pharmacognosy, Manipal College of Pharmaceutical Sciences, Manipal.

\section{Preparation of aqueous extract of GG and NI:}

The roots of GG and leaves of NI were shade dried for seven days and then powdered. Aqueous extraction was carried out by hot maceration method (9).

Experimental animals: Healthy adult Wistar albino rats of either sex, weighing 150-200 g, housed under standard environmental conditions of temperature and humidity $\left(25 \pm 0.5^{\circ} \mathrm{C}\right)$ and 12 hours' light/dark cycle were used in the study. The animals were on a standard pellet diet and water ad libitum. The experimental study was carried out in the central animal house of Kasturba Medical College, Manipal, India, after procurement of the approval from the Institutional Animal Ethics Committee (Ref. No: IAEC/KMC/49/2013).

Wound model and treatment groups: Wistar rats were used for excision wounds. The animals were classified into seven groups (6 animals in each group): Control (untreated), Honey, Ghee, $\mathrm{GG}$, NI, Honey+Ghee $(\mathrm{H}+\mathrm{G})$, a combination of all test materials (Tot).

Creation of wound: The rats were anesthetized with ketamine, before the infliction of the excision wounds. The dorsal fur of the animal was shaved, and the area of the wound to be created was outlined on the back of the animal with methylene blue using a circular stainless steel stencil. A full thickness of the excision wound of $2.5 \mathrm{~cm}$ in width (circular area $=4.90 \mathrm{~cm}^{2}$ ) and 0.2 $\mathrm{cm}$ in depth was created along the markings using toothed forceps, a surgical blade and pointed scissors. The entire wound was left open.

Treatment plan: The open wounds (covering the whole wound area and the adjacent area) were topically applied with reference dose of test materials i.e. $500 \mathrm{mg}$ once a day. Two subclasses of animals with seven groups each were created and followed up to study the nature of healing for 8 and 16 days respectively.
Biomechanical Analysis

Epithelialization period: The number of days required for fall of the dead tissue remnants (scab) without any residual raw wound was considered as the time taken for epithelialization.

Measurement of the rate of wound closure/healing: The wound area was measured every other day, following the first day of the wounding using a permanent marker and thin paper. The wound marks recorded were scanned, and the area was measured using image analyzer software-Image J. A standardized formula was used to calculate the percentage of wound closure (10).

Also, images of the wound with fixed distance and magnification were captured every alternate day until day 16, and the rate of wound closure was compared with various groups of the study. During the treatment plan, the skin tissue of the excision wound was collected on day 8 and day 16 for histological, biochemical and molecular studies.

Histological evaluation: The skin samples were processed to get the paraffin sections. After obtaining the sections ( $5 \mu \mathrm{m}$ in thickness) of the sample, slides were stained with hematoxylin and eosin ( $\mathrm{H}$ and $\mathrm{E}$ ) to identify the rate of wound healing and epithelialization and quantify them. The presence of skin appendages like hair follicles was also identified. Further, a special stain, i.e., Masson's trichrome was used for assessing the area of healing and vascularity, inflammatory responses, fiber composition and distribution and tissue remodeling. The slides were then analyzed and documented using microscopic images with the help of ImagePro Premier 9.1 (Media Cybernetics, Rockville, USA).

Immunohistochemistry (IHC): Formalin-fixed tissue sections $(5 \mu \mathrm{m}$ in thickness) were attached to the slides pretreated with poly-L-lysine (SigmaAldrich). The sections were deparaffinized with xylene and rehydrated in graded ethanol (100 to $50 \%$ ). Antigen retrieval was done by heating the sections in Tris-EDTA Buffer (0.01 M, pH 6.0) for 30 minutes. The slides were then treated with $0.3 \%(\mathrm{v} / \mathrm{v}) \mathrm{H} 2 \mathrm{O} 2$ in water for five minutes to minimize the activity of endogenous peroxidase. 
The sections were later washed with TBS with $0.025 \%$ Triton X-100 and then blocked with $10 \%$ normal goat serum (HIMEDIA, India) and 1\% BSA in TBS for one hour at room temperature. The sections were then incubated for 12 hours at $4{ }^{\circ} \mathrm{C}$ with Anti-IL1 beta antibody (ab9722, Abcam, UK, 1:1000 dilution) or Anti-alpha smooth muscle Actin antibody (ab5694, Abcam, UK, 1:100 dilution) diluted in TBS with 1\% BSA. After washing with TBS with $0.025 \%$ Triton, sections were incubated with the horseradishperoxidase conjugated goat anti-rabbit secondary antibody (ab6721, Abcam, UK, 1:1000 dilutions) for one hour at room temperature. It was followed by rinsing in TBS and incubation with ImPACT NovaRED Peroxidase (HRP) Substrate (SK-4805, Vector Laboratories, U.S.A) according to the manufacturer's instructions. The slides were then counterstained with haematoxylin (VectorHaematoxylin QS, H-3404, Vector Laboratories, U.S.A), dehydrated, cleared and mounted in Vecta Mount mounting medium (H-5000, Vector Laboratories, U.S.A). The slides were then analyzed and documented using Olympus microscope (BX43), Q-Imaging camera and ImagePro Premier 9.1 software (Media Cybernetics, Rockville, USA). Counting was performed manually in a fixed area at $40 \mathrm{x}$ magnification, and then the ratio of the cell count $/ \mathrm{mm} 2$ granulation tissue was calculated. Color markers were used in the software to avoid confusion. The values were then expressed in Mean \pm SEM.

Statistical Analysis: Results were expressed as mean \pm standard errors of mean (SEM). The data were analyzed using Graph Pad Prism software
(Microsoft, San Diego, CA, USA). One-way ANOVA followed by Dunnett's posthoc test was employed to compare between control and treated groups. P value $\leq 0.05$ was considered statistically significant.

\section{RESULTS}

\section{Biomechanical Analysis}

Epithelialization period: Better healing was indicated by the early closure of the wound area in all the treated groups. Further, the rate of epithelialization was also faster in all the treated groups. It was indicated by the prompt withering of the wound scab compared to control on an average of eighth day of healing. Among the treated groups, GG and Tot showed the best results with earliest withering of the scab tissue on an average of the seventh day of healing.

Measurement of the rate of wound closure/healing: Better wound contraction was also observed in the treated groups (Table 1, Figure 1). A significant increase in the rate of wound contraction was consistently noted in the animals treated with Tot on all the days, i.e., from day 2 to day 16 and $\mathrm{H}+\mathrm{G}$ from day 6 to day 16 respectively compared to the control. Further on day 10, all the treated groups showed significant healing. Additionally, NI significantly increased wound contraction in comparison to control on day six while ghee on day eight. Thus, the excision model evaluated the scar maturation and wound contraction which guided us to show the effectiveness of these traditional medicinal preparations on debrided wounds. 


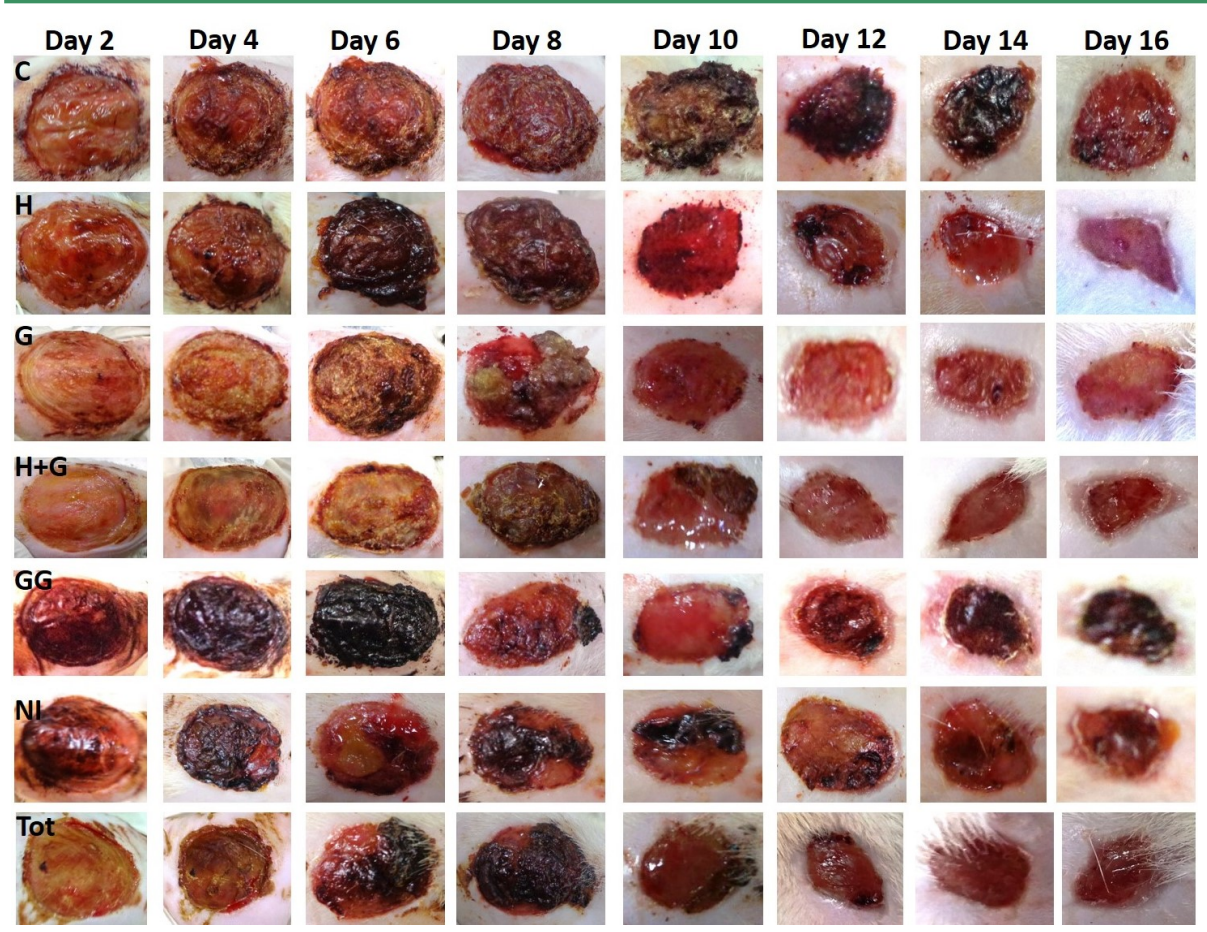

Figure 1: Better and faster healing in all the treated groups of excision model compared to control

Table 1- Effect of topical application of the test materials on the rate of wound closure in excision wound model

\begin{tabular}{|c|c|c|c|c|c|c|c|c|}
\hline \multirow{2}{*}{$\begin{array}{l}\text { Experimen } \\
\text { tal groups }\end{array}$} & \multicolumn{8}{|c|}{ Excision wound model ( $\%$ of wound contraction in days) (Mean \pm SEM) } \\
\hline & 2 & 4 & 6 & 8 & 10 & 12 & 14 & 16 \\
\hline Control & $15.90 \pm 3.77$ & $21.77 \pm 7.01$ & $22.98 \pm 6.65$ & $35.37 \pm 5.11$ & $55.49 \pm 8.47$ & $79.33 \pm 2.65$ & $87.19 \pm 1.69$ & $87.87 \pm 0.59$ \\
\hline Honey & $17.65 \pm 6.13$ & 23.4 & 36.8 & 44 & 74.9 & .14 & $87.78 \pm 2.18$ & 1.84 \\
\hline Ghee & $14.26 \pm 3.74$ & $15.54 \pm 2.95$ & $32.91 \pm 5.85$ & $64.68 \pm 2.78^{\S}$ & $4.92 \pm 2.05^{\S}$ & $86.55 \pm 1.58^{*}$ & $88.90 \pm 0.75$ & $91.04 \pm 2.06$ \\
\hline $\mathrm{H}+\mathrm{G}$ & $16.43 \pm 3.23$ & $29.68 \pm 3.68$ & $6.58 \pm 1.97 *$ & $63.01 \pm 3.13^{\S}$ & ${ }^{\prime} 8.71 \pm 1.84^{\S}$ & $88.75 \pm 2.06^{\S}$ & $2.19 \pm 0.86^{*}$ & $93.89 \pm 0.56^{\S}$ \\
\hline GG & 16.67 & $24.13 \pm 1.91$ & $35.29 \pm 3.97$ & $51.81 \pm 3.64$ & $19.46 \pm 1.35^{\S}$ & $86.19 \pm 0.49$ & $90.35 \pm 0.95$ & $92.22 \pm 1.41$ \\
\hline NI & $11.76 \pm 2.52$ & $20.54 \pm 2.34$ & $42.96 \pm 8.38^{*}$ & $41.44 \pm 7.58$ & ${ }^{\prime} 6.26 \pm 2.83^{\S}$ & $82.54 \pm 1.12$ & $85.95 \pm 1.28$ & $90.29 \pm 0.87$ \\
\hline Tot & $34.38 \pm 2.78^{\S}$ & $41.49 \pm 2.67^{\S}$ & $52.02 \pm 3.86^{\S}$ & $68.48 \pm 4.79^{\S}$ & $82.09 \pm 0.84^{\S}$ & $91.19 \pm 0.94^{\S}$ & $94.52 \pm 0.51^{\S}$ & $94.96 \pm 0.25^{\S}$ \\
\hline
\end{tabular}

${ }^{*} \mathrm{p}<0.05$ vs control, ${ }^{\S} \mathrm{p}<0.01$ vs control

\section{Histological Analysis}

The rate of wound healing: All the treated groups showed better healing compared to the control. This was indicated by early epithelialization and wound closure by the connective tissue matrix as early as $8^{\text {th }}$ day of healing (Figure 2a). By day 16, epithelialization was complete in all treated groups. Better tissue remodeling was also observed in all the treated groups compared to the control. This was indicated by the uniformity of the distribution of the connective tissue matrix at the wound site, unlike the control. Interestingly, groups treated with Honey, $\mathrm{H}+\mathrm{G}, \mathrm{GG}$, and Tot also showed the growth of hair follicles at the wound site (Figure $2 b$ ).

Epithelialisation: Rapid epithelialization was observed and was statistically significant in all the treated groups compared to control on $8^{\text {th }}$ day of wound healing as quantified by the image analyzer software (Figure $2 \mathrm{c} \& \mathrm{~d}$ ). The neo-epithelization was measured from the point of demarcation between the normal and the healing skin. Among all the treated groups honey $(\mathrm{H})$, Glycyrrhiza glabra $(\mathrm{GG})$ and $\mathrm{H}+\mathrm{G}$ showed better epithelialization and wound closure compared with other groups.

DOI: http://dx.doi.org/10.4314/ejhs.v28i6.11 


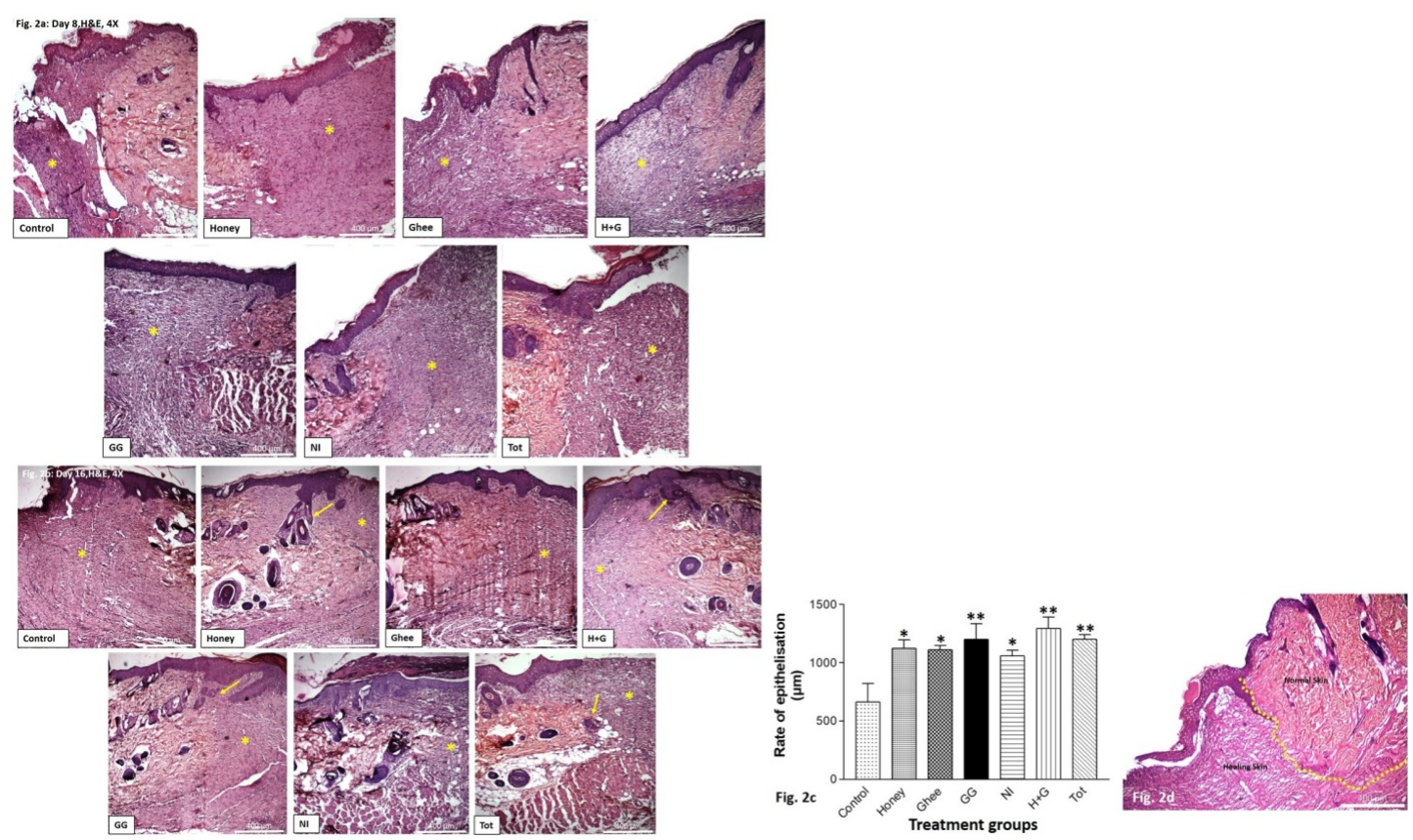

Figure 2a: Haematoxylin and eosin (H\&E) stained sections on the 8th day of treatment at $4 X$ magnification showing the area of healing at the site of excision. All the treated groups showed better healing compared to control.

Figure 2b: Haematoxylin and eosin (H\&E) stained sections on the 16th day of treatment at $4 X$ magnification showing the area of healing at the site of excision. Better tissue remodeling was observed in all the treatment groups compared to control. Honey, GG, and Tot also showed the growth of hair follicles (yellow arrow). *indicates the healing at the site of the wound.

Figure 2c: Graphical representation of the rate of epithelialisation as quantified histologically in all the treated groups compared to control. ${ }^{*} p<0.05$ vs control, ${ }^{* *} p<0.01$ vs control.

Figure 2d: Representative image showing the demarcation between the normal and the healing skin (yellow dotted line). Neo-epithelization was measured from the point of demarcation.

Arrangement in the dermis: Increased vascularity was observed in all the treated groups, unlike the control by Day 8 . This was indicated by the blood filled vacuolated spaces (stained red) in the area of the healing dermis. Increased recruitment of the connective tissue cells was also observed compared to the control (Figure 3a). By day 16 , better tissue remodeling was observed in all the treated groups, unlike the control. This was indicated by the uniformity of the distribution of the connective tissue matrix at the wound site. Enhanced collagen deposition and regular rearrangement was observed at the wound site in all the treated groups compared to control. This was affirmed by the interlacement of the collagen fibers (stained green). The presence of hair follicles in groups treated with Honey, $\mathrm{H}+\mathrm{G}, \mathrm{GG}$ and Tot further confirmed complete healing and regeneration of the skin (Figure $3 b$ ). 


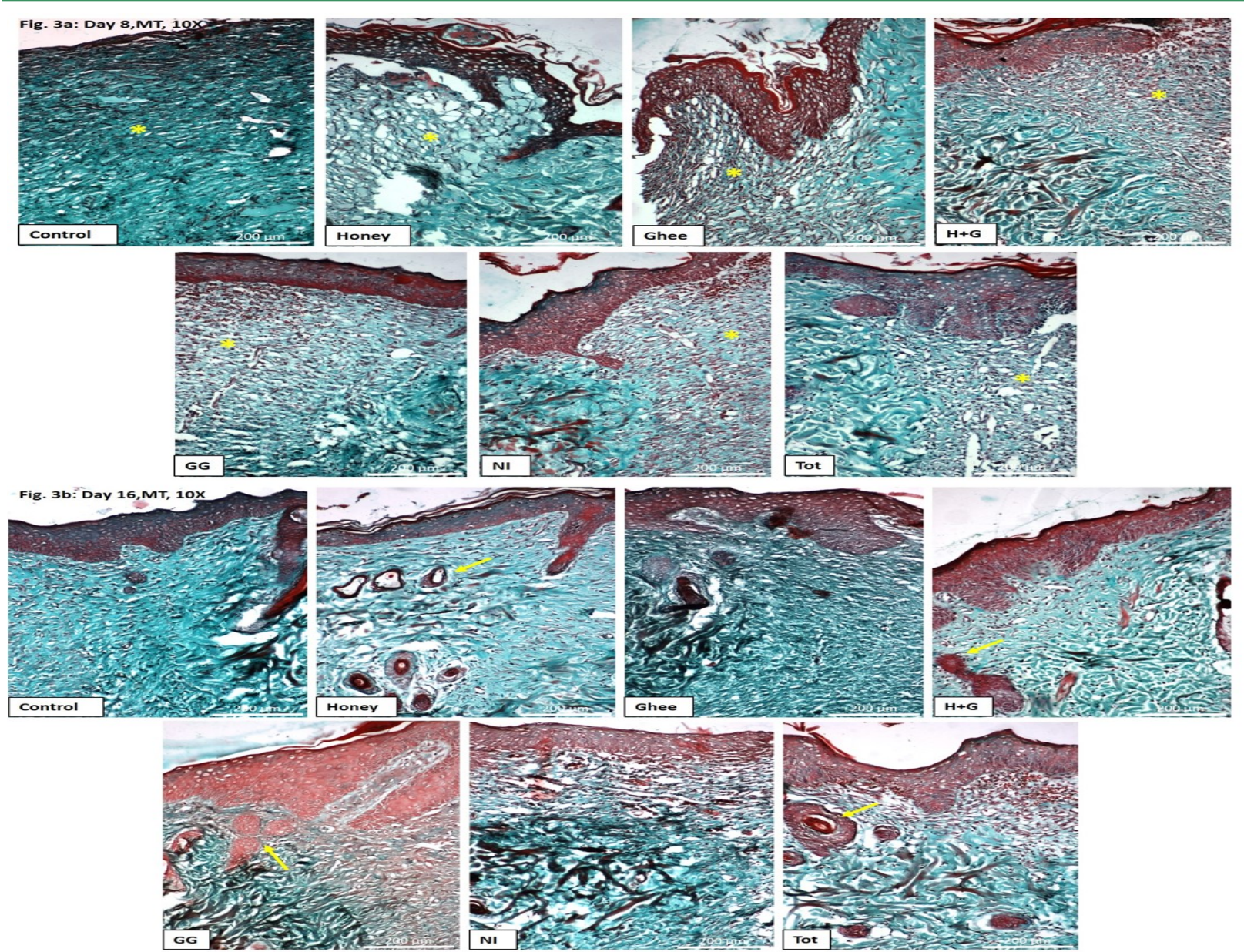

Figure 3a: Masson's trichrome (MT) stained sections on the 8th day of treatment at $10 \mathrm{X}$ magnification showing increased vascularity in all the treated groups unlike control (*indicates the healing site).

Figure 3b: Masson's trichrome (MT) stained sections on the 16th day of treatment at $10 X$ magnification showing enhanced collagen deposition and rearrangement at the wound site in all the treated groups compared to control. Hair follicles were also observed in groups treated with honey, $H+G, G G$, and Tot (Indicated by arrow).

\section{Immunohistochemistry-Horseradish Peroxidase Method (IHC-HRP)}

Inflammation at the wound site by assessing the expression of interleukins (IL1-beta): IL1

beta in the wound tissue is an indicator of inflammatory response usually expressed by the monocytes, macrophages and epithelial cells. Assessment of the activity of IL1 beta in the healing wounds indicated that all the treated groups showed substantial inflammatory activity and better healing (Figure 4).

On day 8, the level of IL1-beta activity was significantly higher in the groups treated with
$\mathrm{H}+\mathrm{G}, \mathrm{GG}, \mathrm{NI}$ and Tot. Honey although showed slightly higher inflammatory activity but was not significant statistically. Ghee showed lower inflammatory activity when compared to control (Figure $4 \mathrm{a} \& \mathrm{c}$ ).

On day 16, all the treated groups showed significantly reduced expression of IL1-beta compared to control. This indicated a reduction in the inflammatory activity and thus better wound healing in all the treated groups unlike the control (Figures $4 \mathrm{~b} \& \mathrm{c}$ ). 

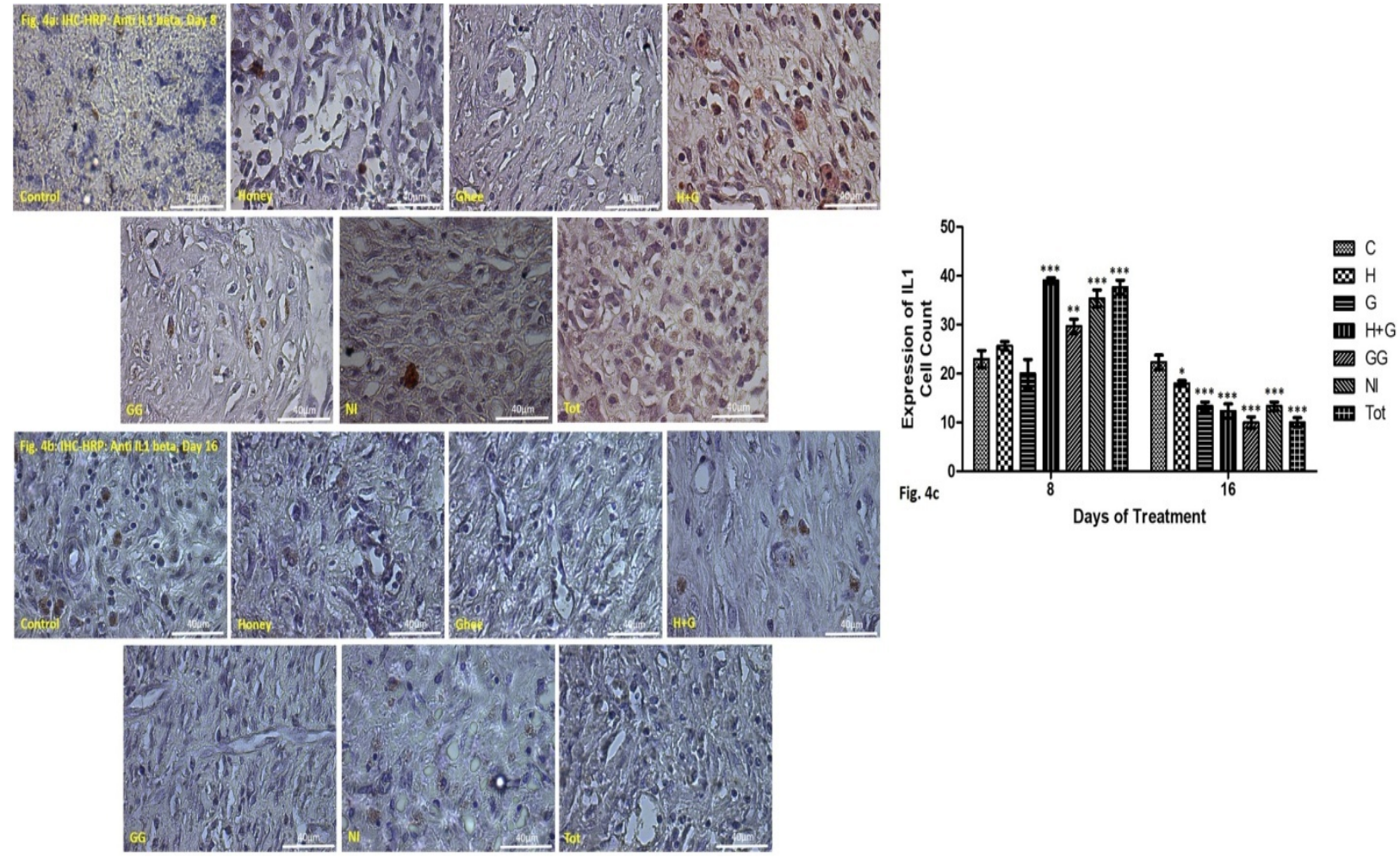

Figure 4a: IHC-HRP staining ILI beta (stained red) in the granulation tissue of the healing rat skin on the $8^{\text {th }}$ day of treatment at $40 X$ magnification.

Figure 4b: IHC-HRP staining ILI beta (stained red) in the granulation tissue of the healing rat skin on the $16^{\text {th }}$ day of treatment at $40 X$ magnification.

Figure 4c: Graphical representation of the cell count showing inflammatory responses (IL1-beta expression) on day $8 \& 16$ in treated groups compared to control. ${ }^{*} p<0.05$ vs control, ${ }^{* *} p<0.01$ vs control, ${ }^{* * *} p<0.001$ vs control.

The activity of myofibroblasts and vascular smooth muscle cells: SMA-positive myofibroblasts were observed in the granulation tissue of the healing skin. SMA reactivity was also intense in the vascular smooth muscle cells indicating increased vascularity (Figure 5).

By day 8 , the activity of the SMA-positive cells was increasingly higher in the groups treated with G, GG and NI both in the granulation tissue and around the sprouting blood vessels compared to the control. The findings were significant statistically. Tot also showed greater SMA positive cell activity compared to the control. The finding was, however, not significant statistically. Groups treated with $\mathrm{H}$ and $\mathrm{H}+\mathrm{G}$ showed relatively lower activity compared to the control (Figures 5a $\& \mathrm{c})$.

On day 16, all the treated groups showed a relatively lower number of SMA positive cell activity compared to the control, indicating better remodeling and healing. Tot although showed lower expression; however, it was not significant statistically (Figures $5 \mathrm{~b} \& \mathrm{c}$ ). 


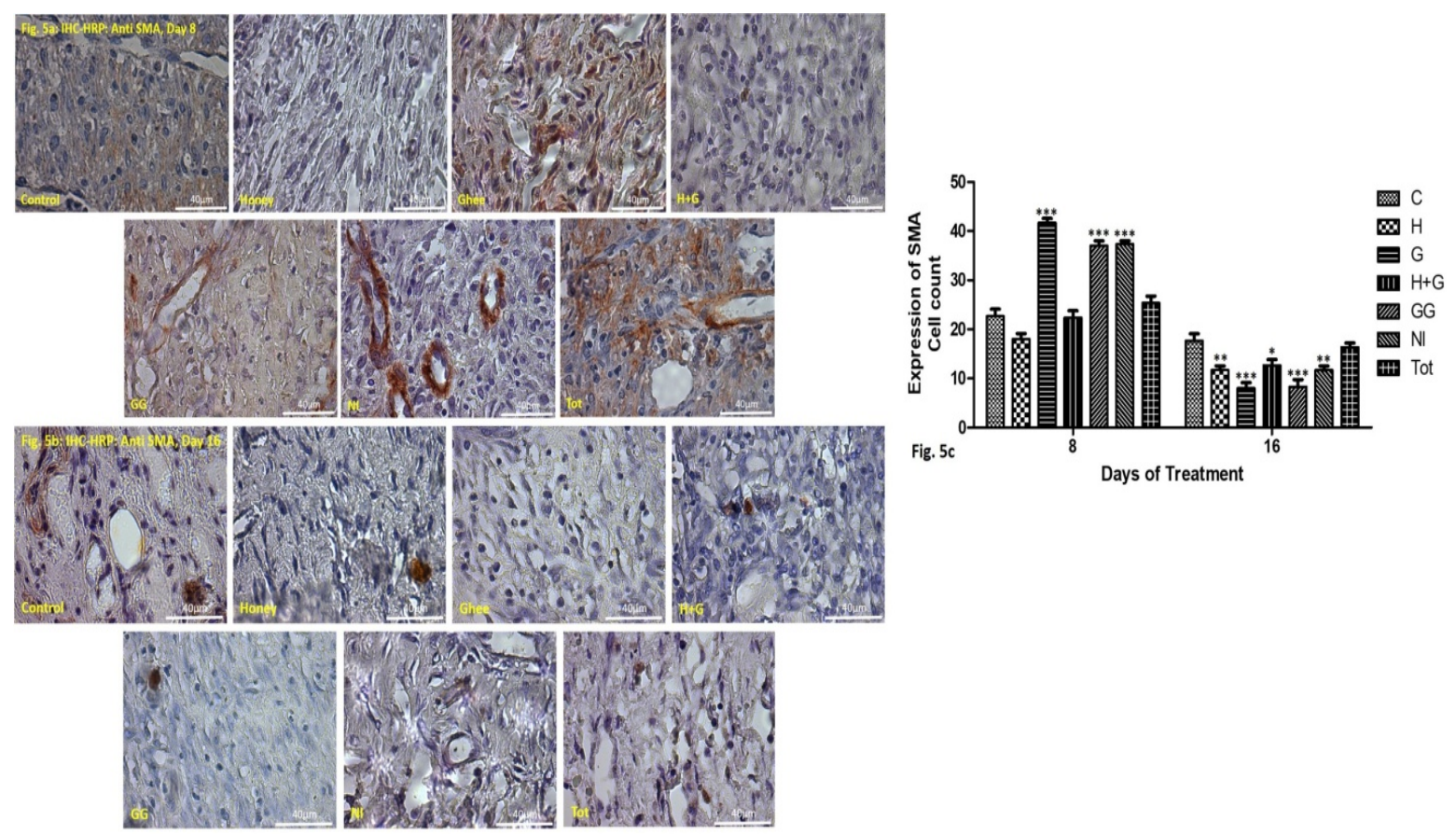

Figure 5a: IHC-HRP staining alpha smooth muscle actin (stained red) in the granulation tissue of the healing rat skin on the $8^{\text {th }}$ day of treatment at $40 X$ magnification.

Figure 5b: IHC-HRP staining alpha smooth muscle actin (stained red) in the granulation tissue of the healing rat skin on the $16^{\text {th }}$ day of treatment at $40 X$ magnification.

Figure 5c: Graphical representation of the cell count showing myofibroblast activity on day 8 \& 16 in treated groups compared to control. ${ }^{*} p<0.05$ vs control, ${ }^{* *} p<0.01$ vs control, ${ }^{* * *} p<0.001$ vs control.

\section{DISCUSSION}

In recent years, it is believed that the polymolecular traditional medicine has more therapeutic benefits than a single molecule based allopathic medicine in wound care. This is because these drugs are not only antiinflammatory and anti-microbial in nature but also has regenerating and rejuvenating effects (11).

Natural medicines like honey, ghee and two plant extracts, i.e., GG and NI are known for their wound healing abilities in Ayurveda and Folk Medicine. Further, their wound healing abilities singly and in combination have been scientifically documented by our previous histological and biomechanical findings in incision wound models (8). However, the precise cellular, biochemical and molecular mechanisms of healing are seldom experimented. This study is, therefore, an attempt to provide an insight into the biomechanical, cellular, biochemical and molecular conditions to healing using excision wounds.
Epithelization and Rate of wound closure: Epithelialization is a major element of wound healing and defines its extent of accomplishment. A wound cannot be considered healed if there is no re-epithelialization.

Benefits of these traditional medicines, singly and in combination, on better epithelization in all the treated groups has been documented by our previous study (8). In this study too, the rate of epithelialization was better in all the treated groups compared to control as indicated by biomechanical and histological findings even in excision wound model. Biomechanical findings showed a faster rate of re-epithelialization in groups like GG and Tot whereas histologically H, $\mathrm{GG}$, and $\mathrm{H}+\mathrm{G}$ showed better findings.

Earlier, it has been shown that a breach in this barrier leads to wound infection and failing to maintain this barrier may pose a significant setback for the process of wound healing (12). Therefore, faster epithelialization by these natural 
medicine, as observed in the present study, is the key for the successful wound healing as these epithelial cells create a barrier between external environment and the wound microenvironment.

Arrangement in the dermis: Normal skin presents a basketlike weave pattern which can never be entirely reproduced with scar remodeling. However, wound remodeling is essential and is closely associated with a great increase in wound-breaking strength (13).

In this present study, better collagen deposition was observed at the wound site in all the treated groups, unlike the control. The arrangement of the collagen was organized and horizontally interlaced in the treated groups. This was unlike the oblique and loose arrangement of the collagen fibers in the control group. Hair follicles were also observed at the wound site in the groups treated with honey, $\mathrm{H}+\mathrm{G}, \mathrm{GG}$ and Tot, further affirming the rejuvenating effects of these groups on wound remodeling. These findings coincide with our previously published report on wound healing in incision wounds (8).

Inflammation at the wound site-by assessing the expression of interleukins (IL1ß): The inflammatory phase is considered to be imperative in the process of repair because it employs the leukocytes that produce growth factors and remove the wound debris. Some inflammatory mediators are up-regulated during the process of healing, like IL-1 (14).

IL-1 is a major pro-inflammatory "alarm" cytokine mainly derived from the macrophages. It enables cell infiltration, inflammation and tissue repair at the site of damage. Of the 11 members of the IL- 1 family of ligands, IL- $1 \alpha$ and IL- $1 \beta$ are the two major agonistic molecules. On the contrary, the IL-1 receptor antagonist (IL-1Ra) is a physiological inhibitor of IL-1. In comparison to IL-1 $\beta$, IL- $1 \alpha$ is less expressed (15). It is, therefore, not considered in this study.

The process of wound healing encompasses a complex relationship between pro-inflammatory and anti-inflammatory cytokines (16). Therefore, it can be postulated that the activity of IL1 $\alpha \& \beta$ are imperative in the early stages of healing by promoting the inflammatory activity. This is then followed by the IL-1Ra signaling which brings down the inflammatory activity and thus becomes critical in the normal wound healing. The findings of this study further justify this fact.

Honey is known to stimulate cytokine production that helps in wound healing (17). However, when the effect of honey on proinflammatory cytokines was previously studied in chronically infected venous leg ulcers, the concentrations of IL-1 were unaffected (16). In this present study, honey treated wounds showed optimal pro-inflammatory activity on day 8 of healing. However, by the end of day 16 , better anti-inflammatory activity was expressed as indicated by lower levels of IL1 $\beta$. This suggests that honey may favour only the required amount of inflammatory activity that is imperative in the early phases of healing. It later takes up the role of an anti-inflammatory agent favouring faster wound healing.

Ghee is also seen to exhibit excellent wound healing property owing to its antimicrobial, immune-stimulant and antioxidant activity $(4,8,18)$. However, its direct influence on the IL1 activity in the wound is less explored. In this study better anti-inflammatory responses of ghee treated wounds were observed compared to the control. The activity of IL-1 $\beta$ was considerably lower by day 8 of healing indicating early inflammatory responses. Honey and ghee in combination showed both pro-inflammatory and anti-inflammatory effects resulting in better healing.

GG (Licorice) has been proved to be beneficial against both acute and chronic inflammatory conditions like rheumatoid arthritis. This was possible by reducing the activity of IL1 $\beta$ (19). However, the anti-inflammatory benefits of GG in wound healing has been less dealt with. In this study, GG treated wounds showed higher levels of IL1 $\beta$ on day 8 which significantly reduced by day 16 . This indicates that GG may regulate the expression of IL1 $\beta$ and exhibits both pro and anti-inflammatory activity which is essential in healing. The pro-inflammatory responses were, however, prolonged in this case indicating continued recruitment of cells to promote wound healing. Similar observations were made in the groups treated with NI and Tot.

DOI: http://dx.doi.org/10.4314/ejhs.v28i6.2 
This may indicate the alternative mode of faster healing that needs to be explored further.

Thus, this study attempted to provide an explanation for the influence of the traditional medicines on the activity of IL-1 signaling in wound healing. It indicated that these traditional medicines possess both pro and anti-inflammatory activity in substantial amounts leading to better healing that may involve different modes of healing process which are not explored extensively.

Role of myofibroblasts in wound healing: Tissue damage activates the fibroblasts and differentiates them into myofibroblasts. These cells create long contractions and actively produce extracellular matrix (ECM) proteins to enable wound closure. Both fibroblasts and myofibroblasts play a critical role in wound healing. These cells, however, show dual effects in the process of wound healing. An appropriate amount of generation of force and deposition of the matrix is advantageous for healing while excessive production of strength and matrix result in tissue scarring and malfunction of repaired tissues (20).

In a study conducted by Haryanto et al., early activity of myofibroblasts were observed in the groups treated with Indonesian Honey in comparison with hydrocolloid dressing i.e., on day 3. Honey thus accelerated the formation of granulation tissue more than the hydrocolloid dressing. On day 7, myofibroblasts activity peaked and was similar in all the groups which then gradually decreased by day 11 and 14 (21). Similar observations were also made by Nakajima et al (22).

In this study, honey and $\mathrm{H}+\mathrm{G}$ failed to show any significant difference in the myofibroblast activity compared to control on day 8 . However, by day 16 , the myofibroblast activity significantly reduced in these groups compared to the control.

The better wound closure has also been observed previously in wounds treated with ghee alone and ghee based formulations $(4,8,18)$. It was attributed to the better activity of myofibroblasts. However, the direct influence of ghee on the activity of myofibroblasts is less discussed in the available literature. In this study,
Ghee treated wounds showed significantly higher activity of myofibroblasts on day 8 . On day 16 , its activity was significantly reduced. This reaffirms the role of ghee in early wound closure without scar formation.

GG has also emerged as a potent wound healer owing to its anti-inflammatory and skin regeneration activity $(6,8)$. Its positive proliferative influence on dermal fibroblasts has also been discussed previously (23). The effect of glycyrrhizin on bleomycin (BLM) induced dermal fibrosis was studied previously. Here, the Glycyrrhizin was found to alleviate dermal fibrosis by significantly reducing the collagen content and number of myofibroblasts (24). In this study, GG treated wounds showed optimal activity of myofibroblasts thereby aiding in better healing. This further supports the fact that GG has a firm control on the activity of the myofibroblasts in wound healing. It increases the myofibroblast activity during the early phases of healing to bring about contraction and wound closure. At the end of the healing phase, GG reduces the myofibroblast activity thereby reducing excessive collagen deposition and thus prevents fibrosis and scar formation.

The biomechanical findings in our previous study had shown increased wound breaking strength in groups treated with NI indicating more tensile strength (8). In this study, NI showed substantial influence on the activity of the myofibroblasts i.e., significantly increased myofibroblast activity on day 8 which decreased significantly by day 16 . It thereby helped in enhancing the wound healing. This further supports the biomechanical findings reported in our previous study. Tot although showed an increase in the activity of myofibroblasts on day 8 and decrease on day 16. This finding, however, was not significant statistically. The findings were better only biologically. This study thus focused on the role of these traditional medicines on the beneficial activity of myofibroblasts in wound healing which has not been discussed previously.

This study, therefore, made an attempt to provide an insight into the biomechanical and cellular, mechanisms of wound healing. The test materials used singly and in combination were

DOI: http://dx.doi.org/10.4314/ejhs.v28i6.11 
beneficial in healing in their unique ways. Further evaluation is required to understand the role of these traditional medicines in treating medically challenging wounds.

\section{REFERENCES}

1. Hunt T, Burke J, Barbul A, Gimble M. Wound healing. Science 1999;284:1775.

2. Robson MC, Steed DL, Franz MG. Wound Healing: Biologic features and approaches to maximize healing trajectories. Curr Probl Surg 2001;38(2):65-140.

3. Jagetia GC, Baliga MS, Venkatesh P, Ulloor JN. Influence of ginger rhizome (Zingiber officinale Rosc.) on survival, glutathione and lipid peroxidation in mice after whole-body exposure to gamma radiation. Radiat Res 2003;160:584-592.

4. Molan PC. The evidence supporting the use of honey as a wound dressing. Int $J$ Lower Extremity Wounds 2006;5:40-54.

5. Prasad V, Dorle AK. Evaluation of ghee based formulation for wound healing activity. $J$ Ethnopharmacol 2006;107:38-47.

6. Oloumi MM, Derakhshanfar A, Nikpoor A. Healing potential of liquorice root extract on dermal wounds in rats. $J$ Vet Res 2007;62:147.

7. Chaitanya SK, Sarvani M, Srilakshmi S, Ashajyothi V. Wound healing herbs-a review. Int J Pharm Technol 2010;2:603-24.

8. Kotian SR, Pai KSR, Nayak JK, Bangera H, Prasad K, Bhat KMR. Biomechanical, Biochemical and Histological Evidences for Wound Healing Properties of Indian Traditional Medicines. Int $J$ Pharm Pharm Sci 2015; 7(11):163-171.

9. Davis H. Bentley's text book of pharmaceutics. 6th edition, Balliere, Tindall and Co: London. 1956: $\mathrm{p}$ 272-30.

10. Lundeberg TCM, Eriksson SV, Malm M. Electrical nerve stimulation improves healing of diabetic ulcers. Ann. Plastic Surg 1992;29:328331.

11. Datta HS, Mitra SK, Patwardhan B. Wound healing activity of topical application forms based on ayurveda. Evid Based Complement Alternat Med, 2011;2011:134378. doi: 10.1093/ecam/nep015.

12. Pastar IP, Stojadinovic O, Yin NC, Ramirez H, Nusbaum A G, Sawaya A, Patel SB, et al. Epithelialization in wound healing: a comprehensive review. Adv Wound Care, 2014; 3(7): 445-464.

13. Monaco JL, Lawrence WT. Acute wound healing. An overview. Clin Plastic Surg, 2003; 30:1- 12.

14. Graves DT, Nooh N, Gillen T, Davey M, Patel S, Cottrell D, Amar S. IL-1 Plays a Critical Role in Oral, But Not Dermal, Wound Healing. $J$ Immunol, 2001;167:5316-5320.

15. Carmi Y, Voronov E, Dotan S, et al. The Role of Macrophage-Derived IL-1 in Induction and Maintenance of Angiogenesis1. J Immunol 2009; 183:4705-4714.

16. Yang CK, Goss S, Alcantara S, et al. Effect of medical honey on pro-inflammatory cytokines in chronically infected venous leg ulcers. Pak $J$ Pharm Sci 2015;28(3):973-81.

17. Tonks AJ, Cooper RA, Jones KP, Blair S, Parton $\mathrm{J}$, Tonks A. Honey stimulates inflammatory cytokine production from monocytes. Cytokine 2003;21:242-247.

18. Fulzele SV, Bhurchandi PM, Kanoje VM, Joshi $\mathrm{SB}$, Dorle AK. Immunostimulant activity of Ashthamangal Ghrita in Rats. Ind J Pharmacol, 2002;34:194-197.

19. Kim KR, Jeong CK, Park KK, Choi JH, Jung Park JHY, Lim SS, Chung WY. Anti-Inflammatory Effects of Licorice and Roasted Licorice Extracts on TPA-Induced Acute Inflammation and Collagen-Induced Arthritis in Mice. $J$ Biomed Biotechnol, 2010;2010:709378. doi: 10.1155/2010/709378. Epub 2010 Mar 17.

20. Li B, Wang JHC. Fibroblasts and Myofibroblasts in Wound Healing: Force Generation and Measurement. J Tissue Viability, 2011; 20(4):108.

21. Haryanto, Urai T, Mukai K, Suriadi, Sugama J, Nakatani T. Effectiveness of Indonesian Honey on the Acceleration of Cutaneous Wound Healing: An Experimental Study in Mice. Wounds, 2012; 24(4):110-119.

22. Nakajima Y, Nakano Y, Fuwano S, et al. Effects of Three Types of Japanese Honey on FullThickness Wound in Mice. Evid Based Complement Alternat Med 2013; 2013:504537. doi: 10.1155/2013/504537.

23. Kotian S R, Padma D, Madhukar R, Bhat KMR. Effect of Natural Medicines on Dermal Fibroblasts in Wound Healing: An In-Vitro Study. Adv Sci Lett, 2017; 23(3):1949-1956.

24. Yamashita $\mathrm{T}$, Asano $\mathrm{Y}$, Taniguchi $\mathrm{T}$, et al. Glycyrrhizin Ameliorates Fibrosis, Vasculopathy, and Inflammation in Animal Models of Systemic Sclerosis. J Invest Dermatol, 2017; 137(3):631.

DOI: http://dx.doi.org/10.4314/ejhs.v28i6.2 